\title{
Randomized phase II study comparing the efficacy and safety of SOX versus mFOLFOX6 as neoadjuvant chemotherapy without radiotherapy for locally advanced rectal cancer (KSCC1301)
}

Keisuke Miwa', Eiji Oki ${ }^{2 *}$ D, Masanobu Enomoto ${ }^{3}$, Keisuke Ihara ${ }^{4}, K_{\text {Koji Ando }}^{2}$, Fumihiko Fujita ${ }^{5}$, Masahiro Tominaga ${ }^{6}$, Shinichiro Mori ${ }^{7}$, Goro Nakayama ${ }^{8}$, Mototsugu Shimokawa ${ }^{9,10}$, Hiroshi Saeki ${ }^{11}$, Hideo Baba ${ }^{12}$, Masaki Mori ${ }^{2}$ and Yoshito Akagi $^{5}$

\begin{abstract}
Background: Preoperative chemoradiotherapy (CRT), the current standard of care for locally advanced rectal cancer (LARC), is associated with many radiotherapy (RT)-related side effects. We aimed to evaluate whether S-1 and oxaliplatin (SOX) or folinic acid, 5-FU, and oxaliplatin (mFOLFOX6) can be as effective as neoadjuvant chemotherapy (NAC) regimens for LARC without RT.
\end{abstract}

Methods: Patients with untreated resectable LARC were randomly assigned to receive SOX or mFOLFOX6. The NAC protocol period was 3 months. The primary endpoint was 3-year disease-free survival (DFS), and the secondary endpoints included pathological effects, surgical completion rate, 3-year survival, and safety.

Results: From September 2013 to October 2015, 56 and 54 patients were enrolled in the SOX and mFOLFOX6 arms, respectively. The 3-year DFS rates were 69.4\% (95\% confidence interval [Cl] 54.9-83.6) and $73.4 \%$ (95\% Cl 58.7-83.6) in the SOX and mFOLFOX6 arms, respectively; no significant differences were found between the arms (log-rank test; $P=0.5315$, hazard ratio: $0.808,95 \% \mathrm{Cl} 0.414-1.578$ ). The 3 -year survival rates were 92.3 and $91.8 \%$ in the SOX and mFOLFOX6 arms, respectively. The surgical completion rate was $98.1 \%$ overall, $100 \%$ in the SOX arm, and $96.0 \%$ in the mFOLFOX6 arm. The incidences of pathological response rates $\geq$ grade $1 \mathrm{~b}$ were 41.5 and $43.8 \%$ in the SOX and mFOLFOX6 arms, respectively. Both treatments were manageable and tolerable.

Conclusion: We demonstrated the effectiveness and safety of SOX and mFOLFOX6, both of which may be new neoadjuvant treatment candidates in previously untreated LARC cases.

(Continued on next page)

\footnotetext{
* Correspondence: okieiji@surg2.med.kyushu-u.ac.jp

${ }^{2}$ Department of Surgery and Science, Graduate School of Medical Sciences, Kyushu University, Maidashi 3-1-1, Higashi-ku, Fukuoka 812-8582, Japan

Full list of author information is available at the end of the article
}

C C The Author(s). 2021 Open Access This article is licensed under a Creative Commons Attribution 4.0 International License, which permits use, sharing, adaptation, distribution and reproduction in any medium or format, as long as you give appropriate credit to the original author(s) and the source, provide a link to the Creative Commons licence, and indicate if changes were made. The images or other third party material in this article are included in the article's Creative Commons licence, unless indicated otherwise in a credit line to the material. If material is not included in the article's Creative Commons licence and your intended use is not permitted by statutory regulation or exceeds the permitted use, you will need to obtain permission directly from the copyright holder. To view a copy of this licence, visit http://creativecommons.org/licenses/by/4.0/. The Creative Commons Public Domain Dedication waiver (http://creativecommons.org/publicdomain/zero/1.0/) applies to the data made available in this article, unless otherwise stated in a credit line to the data. 
(Continued from previous page)

Trial registration: Date of enrolment of the first participant to the trial: 3rd Oct 2013; This study was registered in the UMIN clinical trials registry on 14th Aug, 2013. (Prospectively registered, UMIN-CTR number UMIN000011486). https://upload.umin.ac.jp/cgi-open-bin/ctr/ctr.cgi?function=brows\&recptno=R000013441\&language=J

Keywords: Rectal cancer, Neoadjuvant chemotherapy, Chemoradiotherapy, SOX, mFOLFOX6

\section{Background}

The incidence of colorectal cancer (CRC) is high worldwide, and the disease is third commonly occurring cancer of the United States, with rectal cancer accounting for approximately $30 \%$ of all CRCs [1]. Western countries have employed various treatment approaches for rectal cancer. The Swedish Rectal Cancer Trial showed significantly longer survival durations in association with preoperative short course radiation therapy (RT) than surgery alone [2, 3]. In a meta-analysis performed by Camma et al., who compared surgery alone with preoperative RT, the latter was associated with significantly prolonged survival durations and higher cancer-specific survival rates and significantly reduced local recurrence rates [4]. The efficacies of preoperative RT and preoperative chemoradiotherapy (CRT) were compared and reviewed in the EORTC trial 22,921, [5] FFCD 9203 trial, [6] and Polish 9203 trial [7]. Although none of the aforementioned studies showed that preoperative CRT was superior in terms of survival, the degree of downsizing, downstaging, and histological changes was significantly stronger after preoperative CRT, accompanied by a significant decrease in the rate of local recurrence. Based on the results of those clinical studies, Western guidelines recommend the use of preoperative RT or CRT for T3/T4 or $\mathrm{N}+$ middle or low rectal cancer $[8,9]$. However, although these preoperative treatments significantly improve local recurrence rates, no improvements in survival have been observed [10-13]. Bosset et al. reported 5 year local recurrence rates of 10.9 and $10.7 \%$ and 5 year distant recurrence rates of 32.1 and $29.8 \%$ in the CRT and CRT + adjuvant chemotherapy arms, respectively [14]. These results suggest that the suppression of distant metastasis is important for improving the survival of patients with locally advanced rectal cancer (LARC). Therefore, recently, the total neoadjuvant therapy (TNT) - characterized by the addition of systemic chemotherapy before or after CRT-has been focused to enhance the effectiveness of perioperative treatment $[8,15]$. However, AEs, such as intestinal dysfunction, defecation dysfunction, sexual dysfunction, and secondary cancer occurrence, have been reported after preoperative RT. $[16,17]$ The toxicities caused by $\mathrm{RT}$ remain important concerns in such settings. A recent phase III trial examined the potential for the elimination of RT due to the effects of preoperative chemotherapy [18].
Several studies focusing on the use of preoperative chemotherapy with oxaliplatin-related regimens for rectal cancer have reported high radical resection rates (84-100\%) [19-21]. Although the elimination of RT is expected to reduce the adverse event rates, there is a lack of sufficient data on the related outcomes. In particular, the use of preoperative chemotherapy, including $\mathrm{S}-1$, an oral fluorinated pyrimidine for LARC, has not been investigated so far. In many clinical trials, chemotherapy using S-1 has demonstrated therapeutic results that are similar to those of chemotherapy using 5fluorouracil (5-FU) for metastatic CRC [22-24]. We consider S-1 to be a fluorinated pyrimidine alternative to 5-FU with respect to patient preference and convenience. Therefore, we conducted a multicenter randomized phase II trial (KSCC1301) to evaluate whether S-1 and oxaliplatin (SOX) or folinic acid, 5-FU, and oxaliplatin (mFOLFOX6) are effective neoadjuvant chemotherapy (NAC) regimens without RT for LARC.

\section{Methods \\ Patients}

This trial adhered to the consort statement. This is a multicenter, open-label, randomized phase II trial. The inclusion criteria were as follows: presence of rectal or anal cancer with a low tumor border below the peritoneal reflection; histologically proven rectal anal adenocarcinoma; clinical T3-4 stage disease; clinical N0-2 disease; and M0 (TNM classification, 7th edition, 2010); however, the lymph node in the inferior mesenteric artery region was defined as N3 according to the Japanese Classification of Colorectal Carcinoma [25]; age $\geq 20$ years; Eastern Cooperative Oncology Group performance status score $0-1$; no prior chemotherapy or RT; ability to take medicine; and presence of adequate organ function. Patients were ineligible for participation if they had other primary tumors within the previous 5 years; serious cardiac disease; neurological disease; and renal, hepatic, or bone marrow dysfunction.

Signed informed consent form was obtained from all patients before enrollment to this study. The scientific and ethical aspects of the study were reviewed and approved by the institutional review board (IRB) in each participating institution. The study was conducted according to the principles of the Declaration of Helsinki, and was registered in the UMIN Clinical Trials Registry (UMIN000011486). 


\section{Randomization and masking}

Patients were assigned randomly to (1:1) SOX or mFOLFOX6 arm at the data center of the Clinical Research Support Center Kyushu using the minimization method. Stratification factors were lymph node metastasis (N0 versus $\mathrm{N} 1$ and $\mathrm{N} 2$ ), depth of invasion (T3 versus $\mathrm{T} 4$ ) and institution. Of the series of treatments for LARC, including NAC, surgery, and adjuvant chemotherapy, the protocol treatment in this study was NAC. All patients received the protocol treatment within 14 days of enrollment in the study.

\section{Procedure}

In the SOX arm, S-1 + oxaliplatin was administered every 3 weeks $\left(130 \mathrm{mg} / \mathrm{m}^{2}\right.$ oxaliplatin on day 1 and 80 $120 \mathrm{mg}$ oral S-1 on days $1-14)$. S-1 was administered orally at dosages according to the body surface area (BSA) $\left(120 \mathrm{mg} /\right.$ day for $B S A \geq 1.50 \mathrm{~m}^{2} ; 100 \mathrm{mg} /$ day for BSA $1.25-1.50 \mathrm{~m}^{2} ; 80 \mathrm{mg} /$ day for BSA $<1.25 \mathrm{~m}^{2}$ ). The dosage was divided into two daily doses after meals. In the mFOLFOX6 arm, 5-FU and oxaliplatin were administered every 2 weeks $\left(85 \mathrm{mg} / \mathrm{m}^{2}\right.$ oxaliplatin, $200 \mathrm{mg} / \mathrm{m}^{2}$ leucovorin on day 1 , followed by a $400 \mathrm{mg} / \mathrm{m}^{2}$ bolus of 5-FU and a $46-\mathrm{h} 2400 \mathrm{mg} / \mathrm{m}^{2}$ 5-FU infusion).

Before surgery, patients were scheduled to receive four cycles in the SOX arm and six cycles in the mFOLFOX6 arm. However, imaging tests were performed after two cycles in the SOX arm and three cycles in the mFOLFOX6 arm for the evaluation of whether the tumor had grown and whether radical resection was possible. If chemotherapy could be continued, two cycles were added to the SOX arm and three cycles to the mFOLFOX6 arm. Toxicity was evaluated before the start of each cycle, according to the National Cancer Institute Common Terminology Criteria (NCI-CTC) for Adverse Events version 4.0.

After NAC, radical resection was finally evaluated by performing imaging tests, and if surgery was possible, total mesorectal excision (TME) with lymph node dissection was performed. Regarding the implementation of lateral lymph node dissection, the participating facilities made decisions based on their own criteria. Postoperative adjuvant chemotherapy was started within 8 weeks using the same regimen as preoperative chemotherapy with four cycles in the SOX arm and six cycles in the mFOLFOX6 arm. After completing adjuvant chemotherapy, surveillance was conducted to check for recurrence.

\section{Statistical analysis}

Three-year disease-free survival (DFS) was the primary endpoint. The secondary endpoints were pathological response rate, surgical completion rate, overall survival (OS) rate, and safety. This study assessed the efficacy and safety of NAC without RT using SOX or mFOLFOX6 for LARC and assessed comprehensively selected candidates in a phase III study. In the primary analysis, the 3-year DFS point estimates for the two treatments were calculated, and if one exceeded the other by more than $10 \%$, it was determined to be a promising treatment. However, if the difference was lower than $10 \%$, a decision was made about the right treatment option, which was deemed more likely to succeed by taking the toxicity of both into consideration. In this study, the 3-year DFS was set at $65 \%$ on the basis of previous large-scale clinical trials of preoperative RT for rectal cancer [5-7] and comprehensive evaluation of the treatment results at the participating facilities. To ensure that the probability of the accurate selection of the better treatment arm was $\geq 85 \%$ when the 3 -year DFS exceeded $65 \%$ by at least $10 \%$, the number of cases required was 46 for each arm, using Simon's selection design. Finally, considering some withdrawals and cases that were deemed ineligible to receive preoperative chemotherapy, the target sample size was set as 110 cases, 55 cases in each arm. The registration period was 3 years, and the follow-up period was 5 years. DFS was defined as the duration from the date of surgery to the diagnosis of recurrence or any cause of death or the occurrence of a secondary cancer, whichever occurred first. OS was defined as the duration from the date of surgery until death of any cause. DFS and OS curves were created using the Kaplan-Meier method, and the 95\% confidence intervals (CIs) were estimated using Greenwood's formula. For primary endpoint, a one-sided $p$ value less than 0.05 was considered significant in statistical tests. Statistical analyses were done by SAS version 9.4 software (SAS Institute, Cary, NC).

\section{Results}

Overall, 110 patients were enrolled at 39 sites from September 2013 to October 2015. Although 56 and 54 patients were enrolled in the SOX and MFOLFOX6 arms, respectively, 7 patients did not meet the eligibility criteria. In total, 53 patients in the SOX arm and 50 in the mFOLFOX6 arm received NAC. Therefore, 103 patients were included in the primary endpoint analysis as the full analysis set (Fig. 1). All cases were lower rectal adenocarcinoma, and no case of anal adenocarcinoma was enrolled. The patients' baseline characteristics at the time of registration were well balanced (Table 1). The data cutoff point was October 2018, and median followup for DFS, which was the primary endpoint, was 43.3 months (range, 4.2-58.3 months).

The 3-year DFS rates $69.4 \%$ (95\% CI, 54.9-83.6) and 73.4\% (95\% CI, 58.7-83.6) in the SOX and mFOLFOX6 arms, respectively. A significant difference was not found between the two arms (log-rank test; $p=0.5315$; hazard ratio [HR], 0.808; 95\% CI, 0.414-1.578) (Fig. 2a). The difference in the DFS rate between the SOX and mFOLFOX6 


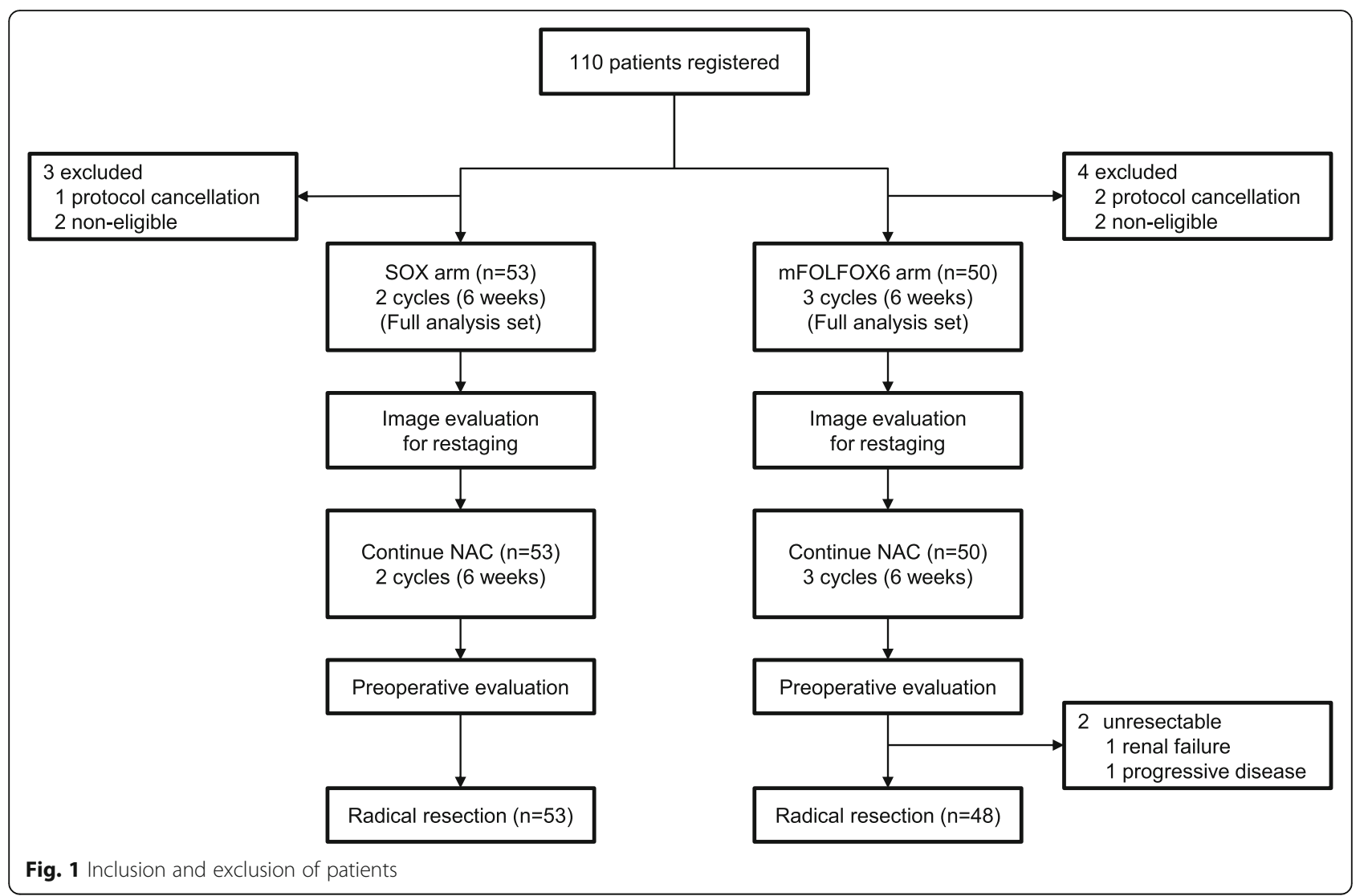

arms was $4.0 \%$ (less than 10\%). The 3-year survival rates for the secondary endpoint were $92.3 \%$ (95\% CI, 80.7-97.0) and 91.8\% (95\% CI, 79.8-96.9) in the SOX and mFOLFOX6 arms, respectively. No significant difference was found between the two arms (log-rank test; $p=0.6897$; HR, 1.307; 95\% CI, 0.350-4.876) (Fig. 2b).

A total of 101 patients underwent TME with lymphadenectomy; 31 of them underwent lateral lymph node dissection. The surgical completion rate was 98.1\% (103 of 105) overall, $100 \%$ (53 of 53) in the SOX arm, and $96.0 \%$ in the mFOLFOX6 arm (48 of 50). Of the two cases in which radical resection failed, one was assessed as unresectable disease on the basis of second preoperative imaging, and the other was considered as being difficult to operate because of renal failure. The incidences of a pathological response rate of grade $1 \mathrm{~b}$ or higher of NAC [25] were $41.5 \%$ (22 of 53 ) and $43.8 \%$ (21 of 48 ) in the SOX and mFOLFOX6 arms, respectively (Table 2). There was no significant difference between the regimens (log-rank test; $p=0.7442$ ).

The incidence of AEs of all grades was $100 \%$ in both arms. The incidences of AEs of grade 3 or higher were 29.5 and $34.2 \%$ in the SOX and mFOLFOX6 arms, respectively. The AEs of grade 3 or higher that occurred in the SOX arm are shown in Table 3. The major AEs were thrombocytopenia (18.9\%) and neutropenia (13.2\%). In the mFOLFOX6 arm (Table 4), they were neutropenia (32.0\%) and leukopenia (6.0\%).

The occurrence rates of perioperative complications of grade II or higher according to the Clavien-Dindo classification [26] were 37.7 and $18.6 \%$ in the SOX and mFOLFOX6 arms, respectively (Table 5). In particular, the SOX arm had a larger number of infection-related complications, including intestinal anastomotic leakage, intra-abdominal abscess, and wound infection, than the mFOLFOX6 arm. No grade IV or higher complications according to the Clavien-Dindo classification were observed in both arms. Finally, 44 (83.0\%) and 38 (79.2\%) patients in the SOX and mFOLFOX6 arms, respectively, received postoperative adjuvant chemotherapy.

\section{Discussion}

In the present study, we found that SOX and mFOLFOX6 as NAC without RT are effective and safe in LARC settings. Regarding NAC, we selected an oxaliplatin-based regimen that is effective against metastatic CRC and reportedly has a high $\mathrm{R} 0$ resection rate even in preoperative chemotherapy. Specifically, for convenience, we decided to compare the mFOLFOX6 regimen, widely used in metastatic CRC, with the SOX regimen, containing oral fluorinated pyrimidine. Although SOX and mFOLFOX6 have been demonstrated to show similar therapeutic 
Table 1 Patient characteristics

\begin{tabular}{|c|c|c|c|}
\hline Characteristics & $\begin{array}{l}\text { SOX arm } \\
n=53(\%)\end{array}$ & $\begin{array}{l}\text { mFOLFOX6 arm } \\
n=50(\%)\end{array}$ & $p$-value \\
\hline \multicolumn{4}{|l|}{ Age (years) } \\
\hline Median (range) & $63(37-79)$ & $64(36-79)$ & 0.3440 \\
\hline \multicolumn{4}{|l|}{ Sex } \\
\hline Male & $44(83.0)$ & $40(80.0)$ & \multirow[t]{2}{*}{0.6930} \\
\hline Female & $9(17.0)$ & $10(20.0)$ & \\
\hline \multicolumn{4}{|c|}{ Performance status (ECOG) } \\
\hline 0 & $53(100.0)$ & $48(96.0)$ & \multirow[t]{2}{*}{0.2332} \\
\hline 1 & $0(0.0)$ & $2(4.0)$ & \\
\hline \multicolumn{4}{|l|}{ Depth of wall invasion } \\
\hline $\mathrm{T} 3$ & $39(73.6)$ & $37(74.0)$ & \multirow[t]{2}{*}{0.9618} \\
\hline $\mathrm{T} 4$ & $14(26.4)$ & $13(26.0)$ & \\
\hline \multicolumn{4}{|l|}{ Lymph node metastasis } \\
\hline No & $20(37.7)$ & $16(32.0)$ & \multirow[t]{4}{*}{0.6317} \\
\hline N1 & $20(37.7)$ & $20(40.0)$ & \\
\hline N2 & $13(24.5)$ & $12(24.0)$ & \\
\hline$N 3^{\mathrm{a}}$ & $0(0.0)$ & $2(4.0)$ & \\
\hline \multicolumn{4}{|l|}{ RAS status } \\
\hline Unexamined & $37(70.0)$ & $36(72.0)$ & \multirow[t]{3}{*}{0.5945} \\
\hline Wild & $8(15.1)$ & $11(22.0)$ & \\
\hline Mutant & $8(15.1)$ & $3(6.0)$ & \\
\hline \multicolumn{4}{|l|}{ Past illness/comorbidity } \\
\hline Yes & $23(43.4)$ & $23(46.0)$ & \multirow[t]{6}{*}{1.0000} \\
\hline Cerebral infarction & $2(3.8)$ & $3(6.0)$ & \\
\hline Myocardial infarction & $1(1.9)$ & $0(0.0)$ & \\
\hline Hypertension & $15(28.3)$ & $14(28.0)$ & \\
\hline Diabetes & $8(15.1)$ & $8(16.0)$ & \\
\hline Others & $11(20.8)$ & $11(22.0)$ & \\
\hline
\end{tabular}

aymph node in the inferior mesenteric artery region,

ECOG: Eastern Cooperative Oncology Group, SOX: S-1 and oxaliplatin, mFOLFOX6: folinic acid, 5-FU, and oxaliplatin

effects in metastatic CRC, [24] no study to date has evaluated the therapeutic outcomes of SOX as an NAC regimen for LARC.

In this study, the 3-year DFS rates were 69.4 and $73.4 \%$ in the SOX and mFOLFOX6 arms, respectively. The 3-year DFS rates in a previous pivotal phase III trial using CRT for rectal cancer were $60-70 \%$ based on Kaplan-Meier curves, [5, 7] similar to our findings. In a recent phase III trial (FOWARC trial) conducted in China, the efficacies of infusional 5-FU + RT + TME, mFOLFOX6 + RT + TME, and mFOLFOX6 + TME were compared; the 3-year DFS rates were 72.9, 77.2, and $73.5 \%$, respectively, and the 3 -year OS rates were 91.3, 89.1, and 90.7\%, respectively [27]. In terms of the 3-year DFS rate, which was the primary endpoint, no difference was noted between the three arms, but the pathological complete response rate was significantly higher in the mFOLFOX6 + RT arm [28]. In our study, the 3-year OS rates were 92.3 and $91.8 \%$ in the SOX and mFOLFOX6 arms, respectively, and the 3-year DFS and 3 -year OS rates were comparable to those in the FOWARC trial. Therefore, although no difference greater than $10 \%$ was observed between both arms, we considered that the treatment outcomes were satisfactory in both arms. Further follow-up is required in the future.

The overall rate of transition to radical resection in this study was $98.1 \%$ (103 of 105), which is a satisfactory result. According to the results of two Japanese prospective studies reporting the efficacy of capecitabine + oxaliplatin + bevacizumab as NAC for LARC, the surgical completion rates were $92 \%$ (23 of 25) [8] and $84 \%$ (27 of 32) [21]. Although the characteristics of our 


\section{a $\quad$ Disease free survival}

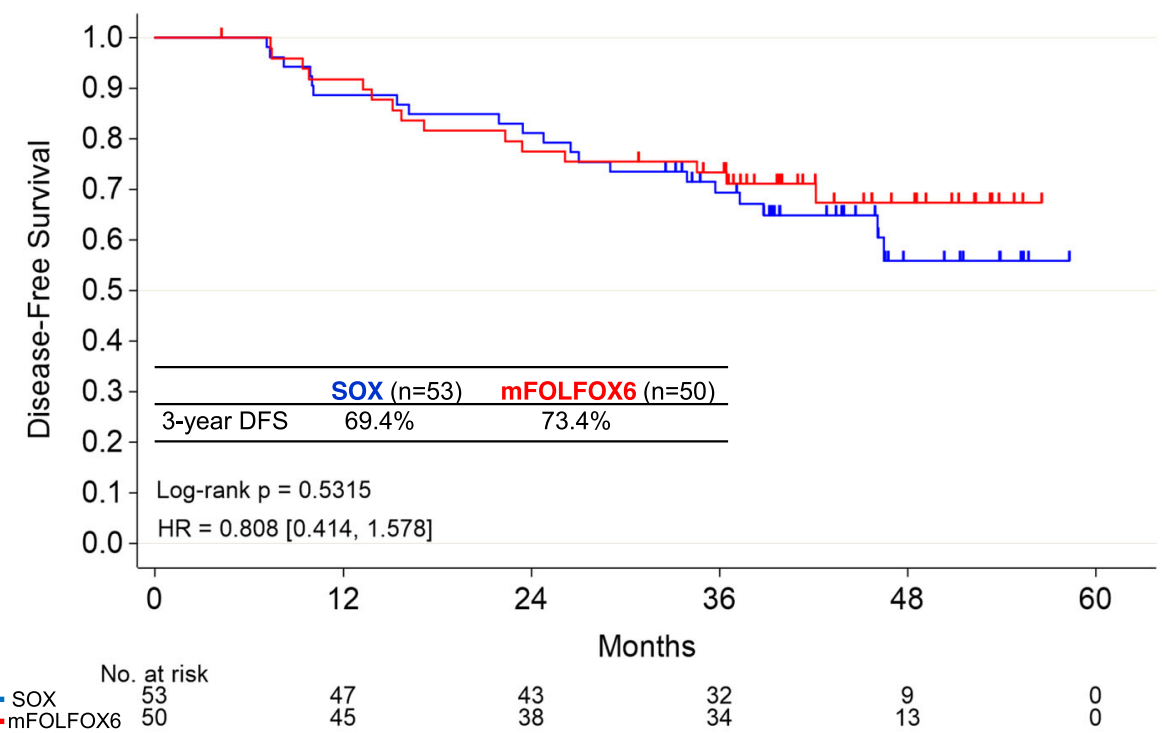

b Overall survival

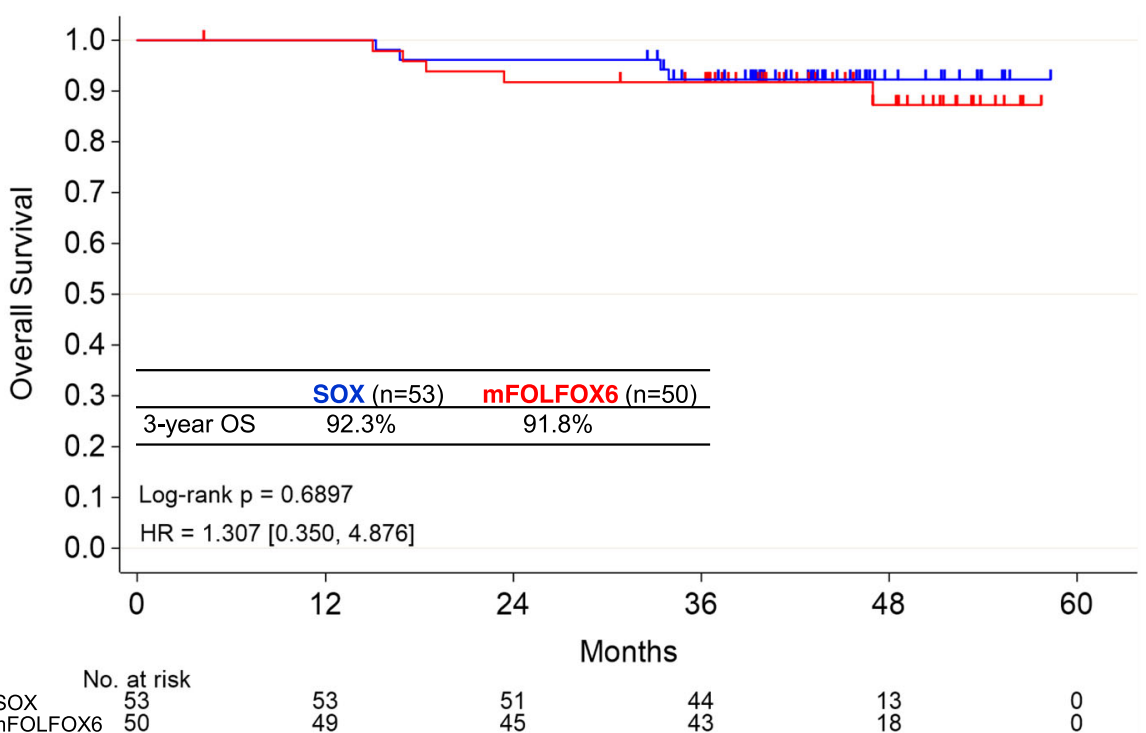

Fig. 2 Disease-free survival (full analysis set) (a) and overall survival (full analysis set) (b)

participants were different, the surgical completion rate in the SOX arm of this study was 100\% (53 of 53), suggesting the effectiveness of SOX as an NAC regimen for LARC. In addition, there was no significant difference in the pathological effectiveness between the regimens, and we believe that it is acceptable to select either as an NAC regimen.

The AEs that occurred in both the regimens were the same as those noted in previously reported clinical phase III trials [29, 30] and are within expectations. Both treatments were manageable and tolerable. In terms of perioperative complications, infection-related complications were more commonly observed in the SOX arm than in the mFOLFOX6 arm, but the cause was unknown. However, complications that were grade IV or higher according to the Clavien-Dindo classification were not observed in both arms. In addition, the transition rate to postoperative adjuvant chemotherapy was comparable between the arms (83.0\% versus 79.2\%). These results indicate the safety of both regimens. 
Table 2 Pathological response rate

\begin{tabular}{llll}
\hline & $\begin{array}{l}\text { SOX arm } \\
\boldsymbol{n = 5 3}(\%)\end{array}$ & $\begin{array}{l}\text { mFOLFOX6 arm } \\
\boldsymbol{n = 4 8}(\%)\end{array}$ & $\begin{array}{l}\text { Total } \\
\boldsymbol{n = 1 0 1}(\%)\end{array}$ \\
\hline Grade 0 & $6(11.3)$ & $5(10.4)$ & $11(10.9)$ \\
Grade 1a & $24(45.3)$ & $22(45.8)$ & $46(45.6)$ \\
Grade 1b & $12(22.6)$ & $8(16.7)$ & $20(19.8)$ \\
Grade 2 & $8(15.1)$ & $12(25.0)$ & $20(19.8)$ \\
Grade 3 & $2(3.8)$ & $1(2.1)$ & $3(3.0)$ \\
Unknown & $1(1.9)$ & $0(0.0)$ & $1(1.0)$ \\
\hline
\end{tabular}

SOX: S-1 and oxaliplatin, mFOLFOX6: folinic acid, 5-FU, and oxaliplatin

In Japan, D3 dissection with lateral lymph nodes, which preserves pelvic autonomic nerve function without preoperative CRT, and subsequent adjuvant chemotherapy are widely used to improve the outcomes of patients with LARC [31]. Mizushima et al. reported that the 3-year DFS rate was $70.1 \%$ in a phase II study of 107 patients with high-risk stage II and stage III rectal cancer without preoperative treatment who had received capecitabine + oxaliplatin therapy as postoperative adjuvant chemotherapy, [32] similar to the findings of our study. There is controversy surrounding whether chemotherapy for LARC should be performed before or after TME in Japan; this issue needs to be resolved in a future phase III study.

Our study has some limitations. First, it had a phase II study design; it is necessary to plan a phase III trial that compares the efficacy of NAC with preoperative CRT or postoperative adjuvant chemotherapy. The standard treatment for LARC is CRT. The regimens used in this study are by no means a substitute for CRT. Second, the incidences of distant metastases and survival were not evaluated because of the insufficient follow-up period. Third, RT was not used. In this study, we selected an RT-free approach to avoid the side effects of RT and to

Table 3 Adverse events $\geq$ grade 3 in the SOX arm ( $n=53$ )

\begin{tabular}{lll}
\hline & $\begin{array}{l}\text { All grades } \\
\mathbf{n}(\%)\end{array}$ & $\begin{array}{l}\text { Grade 3-4 } \\
\mathbf{n}(\%)\end{array}$ \\
\hline Laboratory findings & & \\
Thrombocytopenia & $49(92.5)$ & $10(18.9)$ \\
Neutropenia & $42(79.2)$ & $7(13.2)$ \\
Anemia & $47(88.7)$ & $1(1.9)$ \\
Hypoalbuminemia & $46(86.8)$ & $1(1.9)$ \\
Increased AST & $42(79.2)$ & $1(1.9)$ \\
Hyponatremia & $23(43.4)$ & $1(1.9)$ \\
Clinical findings & & \\
Anorexia & $25(47.2)$ & $2(3.8)$ \\
Paresthesia & $36(67.9)$ & $1(1.9)$ \\
Fatigue/malaise & $21(39.6)$ & $1(1.9)$ \\
\hline AST: aspata
\end{tabular}

AST: aspartate aminotransferase, SOX: S-1 and oxaliplatin
Table 4 Adverse events $\geq$ grade 3 in the mFOLFOX6 arm $(n=50)$

\begin{tabular}{|c|c|c|}
\hline & $\begin{array}{l}\text { All grades } \\
\text { n (\%) }\end{array}$ & $\begin{array}{l}\text { Grade 3-4 } \\
\text { n (\%) }\end{array}$ \\
\hline \multicolumn{3}{|l|}{ Laboratory findings } \\
\hline Neutropenia & $39(78.0)$ & $16(32.0)$ \\
\hline Leukopenia & $28(56.0)$ & $3(6.0)$ \\
\hline Anemia & $41(82.0)$ & $2(4.0)$ \\
\hline Hypoalbuminemia & $44(88.0)$ & $2(4.0)$ \\
\hline Hyponatremia & $18(36.0)$ & $2(4.0)$ \\
\hline Increased AST & $38(76.0)$ & $1(2.0)$ \\
\hline Hypokalemia & $14(28.0)$ & $1(2.0)$ \\
\hline Hyperkalemia & $12(24.0)$ & $1(2.0)$ \\
\hline \multicolumn{3}{|l|}{ Clinical findings } \\
\hline Anorexia & $25(50.0)$ & $2(4.0)$ \\
\hline \multicolumn{3}{|l|}{ Catheter related } \\
\hline Infection & $2(4.0)$ & $2(4.0)$ \\
\hline Fatigue/malaise & $23(46.0)$ & $1(2.0)$ \\
\hline Nausea & $21(42.0)$ & $1(2.0)$ \\
\hline Diarrhea & $21(42.0)$ & $1(2.0)$ \\
\hline Vascular disorder & $1(2.0)$ & $1(2.0)$ \\
\hline Hyperglycemia & $1(2.0)$ & $1(2.0)$ \\
\hline
\end{tabular}

AST: aspartate aminotransferase, mFOLFOX6: folinic acid, 5-FU, and oxaliplatin

Table 5 Perioperative complications $\geq$ grade II per the ClavienDindo classification

\begin{tabular}{|c|c|c|c|}
\hline & $\begin{array}{l}\text { SOX arm } \\
n=53(\%)\end{array}$ & $\begin{array}{l}\text { mFOLFOX6 arm } \\
n=48(\%)\end{array}$ & $\begin{array}{l}\text { Total } \\
n=101 \text { (\%) }\end{array}$ \\
\hline \multicolumn{4}{|c|}{ Postoperative hemorrhage } \\
\hline Illa & $0(0.0)$ & $1(2.1)$ & $1(1.0)$ \\
\hline \multicolumn{4}{|c|}{ Intestinal anastomotic leakage } \\
\hline$\|$ & $1(1.9)$ & $1(2.1)$ & $2(2.0)$ \\
\hline Illa & $1(1.9)$ & $0(0.0)$ & $1(1.0)$ \\
\hline Illb & $4(7.5)$ & $0(0.0)$ & $4(4.0)$ \\
\hline \multicolumn{4}{|c|}{ Intra-abdominal abscess } \\
\hline$\|$ & $1(1.9)$ & $0(0.0)$ & $1(1.0)$ \\
\hline Illa & $3(5.7)$ & $0(0.0)$ & $3(3.0)$ \\
\hline Illb & $2(3.8)$ & $0(0.0)$ & $2(2.0)$ \\
\hline \multicolumn{4}{|c|}{ Wound infection } \\
\hline$\|$ & $4(7.5)$ & $1(2.1)$ & $5(5.0)$ \\
\hline Illa & $2(3.8)$ & $1(2.1)$ & $3(3.0)$ \\
\hline \multicolumn{4}{|l|}{ lleus } \\
\hline Illa & $1(1.9)$ & $2(4.2)$ & $3(3.0)$ \\
\hline Illb & $1(1.9)$ & $2(4.2)$ & $3(3.0)$ \\
\hline \multicolumn{4}{|c|}{ Pneumonia } \\
\hline$\|$ & $0(0.0)$ & $1(2.1)$ & $1(1.0)$ \\
\hline
\end{tabular}

SOX: S-1 and oxaliplatin, mFOLFOX6: folinic acid, 5-FU, and oxaliplatin 
determine if a recently developed chemotherapy regimen could be used in a study arm compete with future CRTs. Further, the therapeutic efficacy of chemotherapy using these regimens as TNT remains unclear. We believe that these two regimens are worth assessing as TNT for LARC in the future.

This study demonstrated the efficacy of SOX and mFOLFOX6 containing oral fluoropyrimidine as NAC regimens for resectable LARC. Data on NAC without RT for T3-T4, N0-N2 resectable rectal cancer are currently very limited. Therefore, we believe that our study is extremely valuable because it provides data on the use of NAC without RT for rectal cancer.

\section{Conclusion}

SOX and mFOLFOX6 as NAC regimens without RT are effective and safe and may be new neoadjuvant treatment candidates in LARC settings.

\section{Abbreviations \\ CRT: chemoradiotherapy; LARC: locally advanced rectal cancer; SOX: S-1 and oxaliplatin; mFOLFOX6: folinic acid, 5-FU, and oxaliplatin; NAC: neoadjuvant chemotherapy; RT: radiotherapy; DFS : disease-free survival; CRC: colorectal cancer; TNT: total neoadjuvant therapy; NCI-CTC: National Cancer Institute Common Terminology Criteria; TME: total mesorectal excision; \\ Cls: confidence intervals (Cls); AEs: Adverse events}

\section{Acknowledgments}

We express our gratitude to all the patients participated in this randomized study. We are grateful to all the physicians at the participating institutions. We also thank secretaries especially Ms. Sanae Sakamoto for their assistance at the data center

\section{Consent to participate}

Not applicable.

\section{Authors' contributions}

KM and EO drafted the manuscript. EO, MS, HS HB, MM and YA were involved in conception and design. MS and EO were involved in analysis and interpretation of data. ME, KI, KA, FF, MT SM and GN were involved in data collection. All authors contributed in review, revise and approve the manuscript; and agree to be accountable to all aspects of the work.

\section{Funding}

This study was funding from Taiho Pharmaceutical Co. Ltd. under a research contract, and supported by KSCC (Kyushu study group of clinical cancer) as a division of Clinical Study and data center. The funders had no role in the design of the study and collection, analysis, and interpretation of data and in writing the manuscript.

\section{Availability of data and materials}

The datasets used and/or analysed during the current study are available from the corresponding author on reasonable request.

\section{Ethics approval and consent to participate}

This study was conducted in accordance with the Declaration of Helsink and Ethical Guidelines for Clinical Studies in Japan, and the protocol was approved by the institutional review boards of all participating medical institutions; Tokyo Medical University Hospital, Dokkyo Medical University Hospital, Kyushu University Hospital, Kurume University, Hyogo Prefectural Cancer Center, Kagoshima University Hospital, Nagoya University Hospital, Fukuoka City Hospital, Nagasaki University Hospital, Kagoshima Kouseiren Hospital, Kitaharima General Medical Center, Saieikai Karatsu Hospital, Toho University Ohmori Hospital, Kumamoto University, JCHO Hitoyoshi Medical Center, Kyushu Medical Center, Kakizoe Hospital, Oita University Hospital, lizuka Hospital, Okayama Rosai Hospital, St Marianna University School of
Medicine, Sasebo City General Hospital, Aichi Prefectural Cancer Center Hospital, Oita Medical Center, Saieikai Sendai Hospital, Kumamoto Medical Center, Imari Arita Kyouritsu Hospital, Tagawa City Hospital, Nagoya Medical Center, Kobe City Medical Center, Urazoe General Hospital, JCHO Kurume General Hospital, Matsuyama Red Cross Hospital, Fukui Saiseikai Hospital, Amakusa Medical Center Hospital. Patients decided whether or not they would participate in the trial after being given a detailed explanation, and written informed consent was obtained from all patients prior to enrolment.

\section{Consent for publication}

The informed consent form sated that the result would publish after completed the study. Japanese regulation. The consent of the family is not be demanded at article publication by the ethic guideline in Japan.

\section{Competing interests}

Outside the submitted work, Keisuke Miwa received grants and personal fees from Taiho Pharm., Ono Pharm., Chugai Pharm. and Takeda Pharm., grants from Merck Biopharm. and Pfizer Inc. and Tsumura \& Co.; personal fees from Yakult Honsha Co., Ltd., Bristol-Myers Squibb Co. and Novartis Pharma K.K., personal fees from AstraZeneca K.K., Kyowa Kirin Co., Ltd., Nippon Kayaku Co. Ltd. and Eli Lilly.. Outside the submitted work, Eiji Oki received personal fees from Taiho Pharm, Bayer, Chugai Pharm., Takeda Pharm., Ono Pharm., Merck Biopharma., MSD and Yakult Honsha. Outside the submitted work, Goro Nakayama received personal fees from Chugai Pharm., Janssen Pharmaceutical K.K., Yakult Honsha Co. Ltd.. Taiho Pharm., Eli Lilly Japan K.K., Takeda Pharm., and Miyarinsan Pharm.

Mototsugu Shimokawa received personal fees from Sysmex outside the submitted work. The other authors have no conflicts of interest to declare.

\section{Author details}

${ }^{1}$ Multidisciplinary Treatment Cancer Center, Kurume University Hospital, Kurume, Japan. ${ }^{2}$ Department of Surgery and Science, Graduate School of Medical Sciences, Kyushu University, Maidashi 3-1-1, Higashi-ku, Fukuoka 812-8582, Japan. ${ }^{3}$ Gastrointestinal and Pediatric Surgery, Tokyo Medical University, Tokyo, Japan. ${ }^{4}$ First Department of Surgery, Dokkyo Medical University, Tochigi, Japan. ${ }^{5}$ Department of Surgery, Kurume University School of Medicine, Kurume, Japan. 'Department of Gastroenterological Surgery, Hyogo Cancer Center, Nishinomiya, Japan. ${ }^{7}$ Department of Digestive Surgery, Breast and Thyroid Surgery, Kagoshima University, Kagoshima, Japan. ${ }^{8}$ Department of Gastroenterological Surgery, Graduate School of Medicine, Nagoya University, Nagoya, Japan. ${ }^{9}$ Cancer Biostatistics Laboratory, Clinical Research Institute, National Hospital Organization Kyusyu Cancer Center, Fukuoka, Japan. ${ }^{10}$ Department of Biostatistics, Yamaguchi University Graduate School of Medicine, Ube, Japan. ${ }^{11}$ Department of Gastroenterological Surgery, Gunma University Graduate School of Medicine, Maebashi, Japan.

${ }^{12}$ Department of Gastroenterological Surgery, Kumamoto University Graduate School of Medicine, Kumamoto, Japan.

Received: 30 September 2020 Accepted: 23 December 2020 Published online: 05 January 2021

\section{References}

1. Siegel R, Desantis C, Jemal A. Colorectal cancer statistics, 2014. CA Cancer J Clin 2014;64:104-117. https://doi.org/https://doi.org/10.3322/caac.21220.

2. Pahlman L, Glimelius B. Improved survival with preoperative radiotherapy in resectable rectal cancer. N Engl J Med 1997;336:980-987. https://doi.org/ https://doi.org/10.1056/NEJM199704033361402.

3. Folkesson J, Birgisson $H$, Påhlman L, et al. Swedish rectal cancer trial: long lasting benefits from radiotherapy on survival and local recurrence rate. J Clin Oncol 2005;23:5644-5650. https://doi.org/https://doi.org/10.1200/JCO. 2005.08.144.

4. Camma C, Giunta M, Fiorica F, Pagliaro L, Craxì A, Cottone M. Preoperative radiotherapy for resectable rectal cancer: a meta-analysis. JAMA 2000;284: 1008-1015. https://doi.org/https://doi.org/10.1001/jama.284.8.1008.

5. Bosset JF, Collette L, Calais G, et al. Chemotherapy with preoperative radiotherapy in rectal cancer. N Engl J Med 2006;355:1114-1123. https://doi. org/https://doi.org/10.1056/NEJMoa060829.

6. Gerard JP, Conroy T, Bonnetain F, et al. Preoperative radiotherapy with or without concurrent fluorouracil and leucovorin in T3-4 rectal cancers: results of FFCD 9203. J Clin Oncol 2006:24:4620-4625. https://doi.org/https://doi. org/10.1200/JCO.2006.06.7629. 
7. Bujko K, Nowacki MP, Nasierowska-Guttmejer A, Michalski W, Bebenek M, Kryj M. Long-term results of a randomized trial comparing preoperative short-course radiotherapy with preoperative conventionally fractionated chemoradiation for rectal cancer. Br J Surg 2006;93:1215-1223. https://doi. org/https://doi.org/10.1002/bjs.5506.

8. Benson AB, Venook AP, Al-Hawary MM, et al. Rectal Cancer, version 2.2018, NCCN clinical practice guidelines in oncology. J Natl Compr Cancer Netw 2018;16:874-901. https://doi.org/https://doi.org/10.6004/jnccn.2018.0061.

9. Glynne-Jones R, Wyrwicz L, Tiret E, et al. Rectal cancer: ESMO Clinical Practice Guidelines for diagnosis, treatment and follow-up. Ann Oncol. 2017; 28:iv22-iv40. https://doi.org/https://doi.org/10.1093/annonc/mdx224.

10. Kapiteijn E, Marijnen CA, Nagtegaal ID, et al. Preoperative radiotherapy combined with total mesorectal excision for resectable rectal cancer. N Engl J Med 2001;345:638-646. https://doi.org/https://doi.org/10.1056/ NEJMoa010580.

11. Peeters KC, Marijnen CA, Nagtegaal ID, et al. The TME trial after a median follow-up of 6 years: increased local control but no survival benefit in irradiated patients with resectable rectal carcinoma. Ann Surg 2007;246:693701. https://doi.org/https://doi.org/10.1097/01.sla.0000257358.56863.ce

12. Sauer R, Liersch T, Merkel S, et al. Preoperative versus postoperative chemoradiotherapy for locally advanced rectal cancer: results of the German CAO/ARO/AIO-94 randomized phase III trial after a median followup of 11 years. J Clinical Oncol. 2012;30:1926-1933. https://doi.org/https:// doi.org/10.1200/JCO.2011.40.1836

13. van Gijn W, Marijnen CA, Nagtegaal ID, et al. Preoperative radiotherapy combined with total mesorectal excision for resectable rectal cancer: 12 year follow-up of the multicentre, randomised controlled TME trial. Lancet Oncol 2011;12:575-582. https://doi.org/https://doi.org/10.1016/S14702045(11)70097-3.

14. Bosset JF, Calais G, Mineur L, et al. Fluorouracil-based adjuvant chemotherapy after preoperative chemoradiotherapy in rectal cancer: longterm results of the EORTC 22921 randomised study. Lancet Oncol. 2014;15: 184-190. https://doi.org/https://doi.org/10.1016/S1470-2045(13)70599-0.

15. Goodman KA. Total neoadjuvant therapy for rectal cancer. Cancer Radiother 2018;22:459-465. https://doi.org/https://doi.org/10.1016/j.canrad.2018.01.004.

16. Birgisson H, Påhlman L, Gunnarsson U, Glimelius B. Occurrence of second cancers in patients treated with radiotherapy for rectal cancer. J Clin Oncol 2005;23:6126-6131. https://doi.org/https://doi.org/10.1200/JCO.2005.02.543.

17. Peeters KC, van de Velde CJ, Leer JW, et al. Late side effects of short-course preoperative radiotherapy combined with total mesorectal excision for rectal cancer: increased bowel dysfunction in irradiated patients--a Dutch colorectal cancer group study. J Clinical Oncol 2005;23:6199-6206. https:// doi.org/https://doi.org/10.1200/JCO.2005.14.779.

18. Weiser MR, Fichera A, Schrag D, Boughey JC, You YQ. Progress in the PROSPECT trial: precision treatment for rectal cancer? Bull Am Coll Surg. 2015;100:51-2

19. Hasegawa J, Nishimura J, Mizushima T, et al. Neoadjuvant capecitabine and oxaliplatin (XELOX) combined with bevacizumab for high-risk localized rectal cancer. Cancer Chemother Pharmacol 2014;73:1079-1087. https://doi. org/https://doi.org/10.1007/s00280-014-2417-9.

20. Schrag D, Weiser MR, Goodman KA, et al. Neoadjuvant chemotherapy without routine use of radiation therapy for patients with locally advanced rectal cancer: a pilot trial. J Clin Oncol 2014;32:513-518. https://doi.org/ https://doi.org/10.1200/JCO.2013.51.7904.

21. Uehara K, Hiramatsu K, Maeda A, et al. Neoadjuvant oxaliplatin and capecitabine and bevacizumab without radiotherapy for poor-risk rectal cancer: N-SOG 03 phase II trial. Jpn J Clin Oncol 2013;43:964-971. https:// doi.org/https://doi.org/10.1093/jjco/hyt115.

22. Muro K, Boku N, Shimada Y, et al. Irinotecan plus S-1 (IRIS) versus fluorouracil and folinic acid plus irinotecan (FOLFIRI) as second-line chemotherapy for metastatic colorectal cancer: a randomised phase 2/3 non-inferiority study (FIRIS study). Lancet Oncol 2010;11:853-860. https://doi. org/https://doi.org/10.1016/S1470-2045(10)70181-9.

23. Yamada Y, Denda T, Gamoh M, et al. S-1 and irinotecan plus bevacizumab versus mFOLFOX6 or CapeOX plus bevacizumab as first-line treatment in patients with metastatic colorectal cancer (TRICOLORE): a randomized open-label, phase III, noninferiority trial. Ann Oncol 2018;29:624-631. https:// doi.org/https://doi.org/10.1093/annonc/mdx816.

24. Yamada Y, Takahari D, Matsumoto $H$, et al. Leucovorin, fluorouracil, and oxaliplatin plus bevacizumab versus S-1 and oxaliplatin plus bevacizumab in patients with metastatic colorectal cancer (SOFT): an open-label, non- inferiority, randomised phase 3 trial. Lancet Oncol. 2013;14:1278-1286. https://doi.org/https://doi.org/10.1016/S1470-2045(13)70490-X.

25. Japanese Classification of Colorectal, Appendiceal, and Anal Carcinoma: the 3d English Edition [Secondary Publication]. J Anus Rectum Colon. 2019;3: 175-95. https://doi.org/https://doi.org/10.23922/jarc.2019-018.

26. Clavien PA, Barkun J, de Oliveira ML, et al. The Clavien-Dindo classification of surgical complications: five-year experience. Ann Surg 2009;250:187-196. https://doi.org/https://doi.org/10.1097/SLA.0b013e3181b13ca2.

27. Deng Y, Chi P, Lan P, et al. Neoadjuvant modified FOLFOX6 with or without radiation versus fluorouracil plus radiation for locally advanced rectal cancer: final results of the Chinese FOWARC trial. J Clin Oncol 2019:37:3223-3233. https://doi.org/https://doi.org/10.1200/JCO.18.02309.

28. Deng Y, Chi P, Lan P, et al. Modified FOLFOX6 with or without radiation versus fluorouracil and leucovorin with radiation in neoadjuvant treatment of locally advanced rectal cancer: initial results of the Chinese FOWARC multicenter, open-label, randomized three-arm phase III trial. J Clin Oncol 2016;34:3300-3307. https://doi.org/10.1200/JCO.2016.66.6198.

29. Andre T, Boni C, Mounedji-Boudiaf L, et al. Oxaliplatin, fluorouracil, and leucovorin as adjuvant treatment for colon cancer. N Engl J Med 2004;350: 2343-2351. https://doi.org/https://doi.org/10.1056/NEJMoa032709.

30. Hong YS, Park YS, Lim HY, et al. S-1 plus oxaliplatin versus capecitabine plus oxaliplatin for first-line treatment of patients with metastatic colorectal cancer: a randomised, non-inferiority phase 3 trial. Lancet Oncol. 2012;13: 1125-1132. https://doi.org/https://doi.org/10.1016/S1470-2045(12)70363-7.

31. Sugihara K, Kobayashi H, Kato T, et al. Indication and benefit of pelvic sidewall dissection for rectal cancer. Dis Colon Rectum 2006;49:1663-1672. https://doi.org/https://doi.org/10.1007/s10350-006-0714-z.

32. Mizushima T, Ikeda M, Kato T, et al. Postoperative XELOX therapy for patients with curatively resected high-risk stage II and stage III rectal cancer without preoperative chemoradiation: a prospective, multicenter, openlabel, single-arm phase II study. BMC Cancer 2019;19:1-8. https://doi.org/ https://doi.org/10.1186/s12885-019-6122-2.

\section{Publisher's Note}

Springer Nature remains neutral with regard to jurisdictional claims in published maps and institutional affiliations.
Ready to submit your research? Choose BMC and benefit from:

- fast, convenient online submission

- thorough peer review by experienced researchers in your field

- rapid publication on acceptance

- support for research data, including large and complex data types

- gold Open Access which fosters wider collaboration and increased citations

- maximum visibility for your research: over $100 \mathrm{M}$ website views per year

At BMC, research is always in progress.

Learn more biomedcentral.com/submission 